EESTI NSV TEADUSTE AKADEEMIA TOIMETISED. XII KÖIDE FOOSIKA-MATEMAATIKA- JA TEHNIKATEADUSTE SEERIA. 1963, NR. 4

ИЗВЕСТИЯ АКАДЕМИИ НАУК ЭСТОНСКОИ ССР. ТОМ ХІІ СЕРИЯ ФИЗИКО-МАТЕМАТИЧЕСКИХ И ТЕХНИЧЕСКИХ НАУК. 1963, N

\title{
ОБ ИЗМЕНЧИВОСТИ ВОЗМОЖНЫХ ДНЕВНЫХ СУММ ПРЯМОИ РАДИАЦИИ
}

\section{и. УндлА}

Рассматривается зависимость изменчивости возможных дневных сумм прямой солнечной раднацин от колебания эффективной прозрачности атмосферы.

Актинометрическими наблюдениями установлено, что изменчивость возможных дневных сумм прямой радиации, хотя эти суммы соответствуют отсутствию облачности, т. е. отсутствию облаков и туманов, может совершаться в довольно широких пределах. Учет этого вариационного явления помогает повышению точности расчетов сумм прямой радиации и, следовательно, представляет собой задачу довольно большой практической важности.

Изменчивость возможных дневных сумм прямой радиации обусловлена колебаниями эффективной прозрачности атмосферы. Задача предлагаемой работы состоит в том, чтобы более подробно выяснить, как зависит изменчивость возможных дневных сумм прямоӥ радиации от колебания эффективной прозрачности атмосферы и какова при этом роль годового изменения высоты Солнца.

При рассмотрении указанных здесь вопросов мы исходили из возможных дневных сумм прямой радиации, падающей на горизонтальную. поверхность (назовем эти суммы возможными дневными суммами инсоляции). В основу анализа взяты относительные возможные дневные суммы инсоляции $\left(\alpha_{6}^{\prime}\right)$, т. е. $\alpha_{6}^{\prime}=\frac{\Sigma S_{6}^{\prime}}{\Sigma S_{0}^{\prime}}$, где $\Sigma S_{6}^{\prime}$ - действительная возможная дневная сумма инсоляции, $\Sigma S_{0}^{\prime}-$ средняя сглаженная возможная дневная сумма (она имеет постоянное значение для данного календарного дня, т. е. в данном географическом пункте).

Следует отметить, что исследование вариаций значений $\alpha_{\sigma}^{\prime}$ является эквивалентным исследованию колебания возможных дневных сумм прямой радиации, падающей на перпендикулярную к солнечным лучам поверхность, так как $\frac{\Sigma S_{6}^{\prime}}{\Sigma S_{0}^{\prime}}=\frac{\Sigma S_{6}}{\Sigma S_{0}}$, где $\Sigma S_{6} \quad$ и $\Sigma S_{0}-$ соответствующие абсолютные суммы на перпендикулярную поверхность.

Возможную дневную сумму инсоляции можно выразить, исходя из формулы Буге, в следующем виде:

$$
\Sigma S_{6}^{\prime}=\frac{I_{0}}{r^{2}} \int_{i_{\mathrm{B}}}^{t_{3}} p_{m}^{m} \sin h_{\odot} d t
$$


где $I_{0}-$ солнечная постоянная,

$r$ - расстояние между Землей и Солнцем, выраженное в астрономических единицах,

$m$ - относительная длина пути, проходимая солнечными лучами в атмосфере, т. е. так называемая масса атмосферы,

$p_{m}$ - интегральный коэффициент прозрачности атмосферы,

$h_{\odot}$ - высота Солнца,

$t$ - время $\left(t_{\mathrm{B}}-\right.$ момент восхода, $t_{3}-$ момент захода Солнца).

Но подинтегральную функцию, входящую в формулу (1), можно выразить через эффективные величины. Такие величины являются какими-то средними; которые не изменяются в течение дня. Можем написать, что

$$
\Sigma S_{6}^{\prime}=\frac{I_{0}}{r^{2}} p_{m_{9}}^{m_{3}} \sin h_{9} \int_{t_{\mathrm{B}}}^{t_{3}^{*}} d t
$$

где $m_{9}$ - эффективная масса атмосферы, $p_{m_{3}}$ - эффективный коэффициент прозрачности атмосферы, $h_{\ni}$ - эффективная высота Солнца. Понятие последних двух эффективных величин является здесь аналогичным понятию эффективной прозрачности, указанному в работах $[1,2]$, но при предположении, что в нсходную формулу вводится значение эффективного коэффициента прозрачности. Выражение $\frac{I_{0}}{r^{2}} p_{m_{9}}^{m_{9}} \sin h_{9}$ пред. ставляет собой среднюю плотность возможных инсоляционных потоков.

Эффективная масса определяется из условия, что по ее значению, подставленному либо в формулу

$$
\Sigma S_{6}^{\prime}=\frac{I_{0}}{r^{2}} \int_{t_{\mathrm{B}}}^{t_{3}} p_{m_{9}}^{m} \sin h_{\odot} d t
$$

либо в формулу

$$
\Sigma S_{6}=\frac{I_{0}}{r^{2}} \int_{t_{\mathrm{B}}}^{t_{3}} p_{m_{9}}^{m} d t
$$

получается правильное значение возможной дневной суммы, соответствующее заданному значению эффективного коэффициента прозрачности. Эффективная высота Солнца определяется аналогично из формулы

$$
\Sigma S_{6}^{\prime}=\frac{I_{0}}{r^{2}} \int_{t_{\mathrm{B}}}^{t_{3}} p_{m_{9}}^{m} \sin h_{\odot} d t .
$$

Но эффективную высоту Солнца можно определить и по эффективной массе атмосферы (возможно также обратное действие).

Обозначим продолжительность дня через $\Delta t$, т. е. $\int_{t_{\mathrm{B}}} d t=\Delta t$, и выразим формулу (2) в виде

$$
\Sigma S_{6}^{\prime}=\frac{I_{0}}{r^{2}} p_{m_{9}}^{m_{9}} \sin h_{9} \Delta t .
$$


Средняя сглаженная возможная дневная сумма, согласно формуле (3), выразится в виде

$$
\Sigma S_{0}^{\prime}=\frac{I_{0}}{r_{(0)}^{2}} p_{m_{9,0}}^{m_{9,0}} \sin h_{9,0} \Delta_{0} t,
$$

где $r_{(0)}, m_{9,0}, p_{m_{9,0}}, h_{9,0}$ и $\Delta_{0} t$ соответствуют значению $\Sigma S_{0}^{\prime}$.

Относительную возможную дневную сумму инсоляции можно выразить теперь через формулы (3) и (4), т. е.

$$
\alpha_{6}^{\prime}=\frac{\frac{I_{0}}{r^{2}} p_{m_{\ni}}^{m_{9}} \sin h_{9} \Delta t}{\frac{I_{0}-p_{9,0}^{m_{3}} \sin h_{9,0} \Delta_{0} t}{r_{(0)}^{2} p_{m_{9}, 0}}} .
$$

Так как в соотношении $\frac{\Sigma S_{6}^{\prime}}{\Sigma S_{0}^{\prime}}$ оба члена относятся к одному и тому же календарному дню, то можно предположить, что в формуле (5) $r=r_{(0)}, m_{9}=m_{9,0}, h_{\ni}=h_{9,0}$ и $\Delta t=\Delta_{0} t$, причем расхождениями, которые могут здесь наблюдаться, можно пренебречь.

Следовательно, можно написать

$$
a_{6}^{\prime}=\left(\frac{p_{m_{9}}}{p_{m_{9,0}}}\right)^{m_{9,0}} .
$$

Отклонение относительной возможной дневной суммы ннсоляции $\left(\Delta \alpha_{6}^{\prime}\right)$ определяется формулой

$$
\Delta a_{6}^{\prime}=a_{6}^{\prime}-1 \text {, }
$$

но принимая во внимание формулу (6), имеем

$$
\Delta \alpha_{6}^{\prime}=\left(\frac{p_{m_{9}}}{p_{m_{9,0}}}\right)^{m_{9,0}}-1 .
$$

Причина колебания относительных возможных дневных сумм инсоляции заключается в колебании относительного эффективного коэффициента прозрачности атмосферы $(\varepsilon)$, т. е. $\varepsilon=\frac{p_{m_{9}}}{p_{m_{9,0}}}$. Вариация названных сумм наблюдается при условии $p_{m_{9}} \neq p_{m_{9}, 0}$, так как в этом случае $a_{6}^{\prime} \neq 1$ и $\Delta \alpha_{6}^{\prime} \neq 0$ (т. е. $\left.\Sigma S_{6}^{\prime} \neq \Sigma S_{0}^{\prime}\right)$. Но при условии $p_{m_{3}}=p_{m_{\jmath, 0}}$ $a_{6}^{\prime}=1$, т. е. $\Delta \alpha_{6}^{\prime}=0$, вариация сумм отсутствует $\left(\Sigma S_{6}^{\prime}=\Sigma S_{0}^{\prime}\right)$.

Амплитуда колебания относительных возможных дневных сумм инсоляции зависит от соотношения $\frac{p_{m_{9}}}{p_{m_{9.0}}}$ и от $m_{9,0}$. Раздельный учет зависимости от $m_{3,0}$ является необходимым особенно при рассмотрении колебания относительных сумм, относящихся к различным эффективным массам атмосферы. Различие рассматриваемых масс обусловлено как годовым, так и географическим изменением $m_{9}$, причем изменчивостью $m_{\ni}$ можно практически пренебречь. 
Нередко прозрачность атмосферы характеризуется при помощи фактора мутности. Из теории известно, что

$$
p_{m}=q_{m}^{T_{m}},
$$

где $T_{m}$ - интегральный фактор мутности, $q_{m}$ - интегральный коэффищиент, характеризующий прозрачность рэлеевской атмосферы.

Переходя к эффективным величинам,

$$
p_{m_{9}}=q_{m_{9}}^{T_{m_{9}}}
$$

где $q_{m_{3}}$ и $T_{m_{9}}-$ эффективные величины, причем при $\Sigma S_{0}^{\prime}$

$$
p_{m_{9,0}}=q_{m_{9,0}}^{T_{m_{3}, 0}}
$$

Относительный эффективный коэффициент прозрачности, исходя из формул (10) и (11), можно выразить в виде

$$
\frac{p_{m_{9}}}{p_{m_{9,0}}}=q_{m_{9,0}}^{T_{m_{9}}-T_{m_{9,0}}},
$$

принимая во внимание, что в данный конкретный день практично $q_{m_{9}}=q_{m_{9,0}}$.

Следовательно, исходя из формул (6) и (12), можно написать

$$
a_{6}^{\prime}=q_{m_{9,0}}^{m_{9,0}\left(T_{m_{9}}-T_{m_{9,0}}\right) .}
$$

Возможные дневные суммы инсоляции взяты в данной работе относительно средних возможных дневных сумм. Но за фоновые суммы могут быть приняты также суммы инсоляции другого устойчивого типа. Такими фоновыми суммами могут быть, например, экстратеррестрические суммы инсоляции [3].

Можно написать, что в какой-то день «i» $\alpha_{6, i}^{\prime}=\varepsilon_{i}^{m_{9}, i}$, а в какой-то день «u» $\alpha_{6, u}^{\prime}=\varepsilon_{u}^{m_{9, u}}$. Понятно, что при одинаковых относительных эффективных коэффициентах прозрачности, отличающихся от единицы, равенство $\boldsymbol{a}_{6, i}^{\prime}=\boldsymbol{a}_{6 u}^{\prime}$ будет выполняться только тогда, когда $m_{3, i}=m_{9, n}$, а в остальных случаях, т. е. при $m_{9, i} \neq m_{9, u}, a_{6, i} \neq a_{6, u}^{\prime}$. Все варианты следующие:

1) если $m_{3, i}<m_{3, u}$, то

3) если $m_{9, i}=m_{9, u}$, то $\boldsymbol{\alpha}_{6, i}^{\prime}=a_{6, u}^{\prime}$;

а) при $\varepsilon<1 \quad a_{6, i}^{\prime}>a_{6, u}^{\prime}$,

б) при $\varepsilon>1 \quad a_{6, i}^{\prime}<a_{6, u}^{\prime}$;

4) если $\varepsilon=1$, то $\alpha_{6, i}^{\prime}=a_{6, u}^{\prime}=1$.

2) если $m_{\ni, i}>m_{3, u}$, то

а) при $\varepsilon<1 \quad a_{6, i}^{\prime}<a_{6, u}^{\prime}$,

б) при $\varepsilon>1 \quad a_{6, i}^{\prime}>a_{6, u}^{\prime}$;

В общем, если $\varepsilon_{i}=\varepsilon_{n}$, то расхождение значения $\alpha_{6, i}^{\prime}$ от значения $\alpha_{6, t}^{\prime}$ должно быть тем больше, чем больше разница между $m_{9, i}$ и $m_{3, u}$.

В качестве чнслового примера приводятся здесь некоторые вычисления, чтобы конкретизировать представление о зависимости значений $\alpha_{6}^{\prime}$ от эффективной массы атмосферы*.

* Bce представленные в работе конкретные примеры соответствуют условням нормального давлення воздуха. 
Предположим, что $p_{m_{3}}=0,70$ и $p_{m_{3,0}}=0,80$. Го проведенным Х. Ю. Мюрком вычислениям найдем, что при солнцестояниях и осеннем равноденствии значениям указанных здесь коэффициентов соответствуют на географической широте Тарту следующие возможные дневные суммы инсоляции:

22. VI 1) если $p_{m_{9}}=0,70$, то $\Sigma S_{6}^{\prime}=520 \cdot \kappa a л / c \mu^{2} \cdot$ день,

2) $\quad, \quad p_{m_{9,0}}=0,80, \quad, \quad \Sigma S_{0}^{\prime}=648$

23. IX 1) , $\quad p_{m_{3}}=0,70, \quad, \quad \Sigma S_{6}^{\prime}=185$

2) $\quad, \quad p_{m_{9,0}}=0,80, \quad, \quad \Sigma S_{0}^{\prime}=255$

22. XII 1) $, \quad p_{m_{9}}=0,70, \quad, \Sigma S_{6}^{\prime}=4$

2) $\quad, \quad \dot{p}_{m_{3,0}}=0,80, \quad, \quad \Sigma S_{0}^{\prime}=10$

Отсюда следует, что указанным здесь конкретным условиям соответствуют следующие относительные суммы:

22. VI $\alpha_{6}^{\prime}=0,80$,

23. IX $a_{\sigma}^{\prime}=0,73$,

22. XII $\alpha_{\sigma}^{\prime}=0,40$.

В рассматриваемом случае

$$
\frac{\alpha_{6,22 . \mathrm{VI}}^{\prime}}{\alpha_{6,22 . \mathrm{XII}}^{\prime}}=2,0, \quad \frac{a_{6,22 . \mathrm{VI}}^{\prime}}{a_{6,23 . \mathrm{IX}}^{\prime}}=1,1 .
$$

Некоторое представление о годовом ходе относительных возможных дневных сумм инсоляции, соответствующих на географической широте Тарту, одинаковым значениям относительного эффективного коэффициента прозрачности атмосферы, получается при рассмотрении графиков, приведенных на рис. 1. Графики построены для $\varepsilon=0,81 ; 0,94 ; 1,00$; 1,06 .

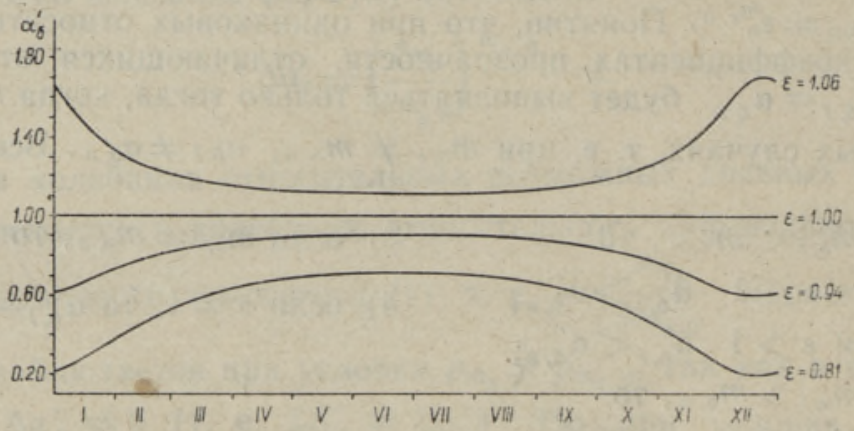

Рнс. 1. Годовой ход относительных возможных дневных сумм инсолящии на географической широте Тарту при $\varepsilon=0,81 ; 0,94 ; 1,00 ; 1,06$.

Из представленных нами примеров выясняется, что величины $\alpha_{\sigma}^{\prime}$ довольно значительно могут зависеть от годового изменения $m_{3}$. Этот факт говорит о том, что сравнение относительных дневных сумм инсоляции, относящихся к различным эффективным массам атмосферы, в общем случае невозможно без приведения относительных сумм к одной и той же массе атмосферы. 
Годовой ход $m_{3}$ зависит практически от годового хода $h_{\mathfrak{3}}$, так как зависимость от годового изменения остальных факторов оказывается небольшой.

Зависимость относительной возможной дневной суммы инсоляции от эффективной прозрачности атмосферы нелинейна, - выражается степенным законом, а в связи с годовым изменением массы атмосферы наблюдается экспоненциальная зависимость от $m_{9}$. Имеется также зависимость от $p_{m_{9,0}}$, так как в течение года этот коэффициент изменяется.

Некоторое иллюстрирующее представление о зависимости значений $a_{6}^{\prime}$ от $p_{m_{9}}$ и от $m_{9}$ дает рис. 2 , причем $p_{9,0}=0,80$. В соответствин с условиями составления графиков, все рассматриваемые кривые пересекаются в одной и той же точке (т. е. $\alpha_{6}^{\prime}=1$ и $\frac{p_{m_{\ni}}}{p_{m_{9,0}^{\prime}}}=1$ ).

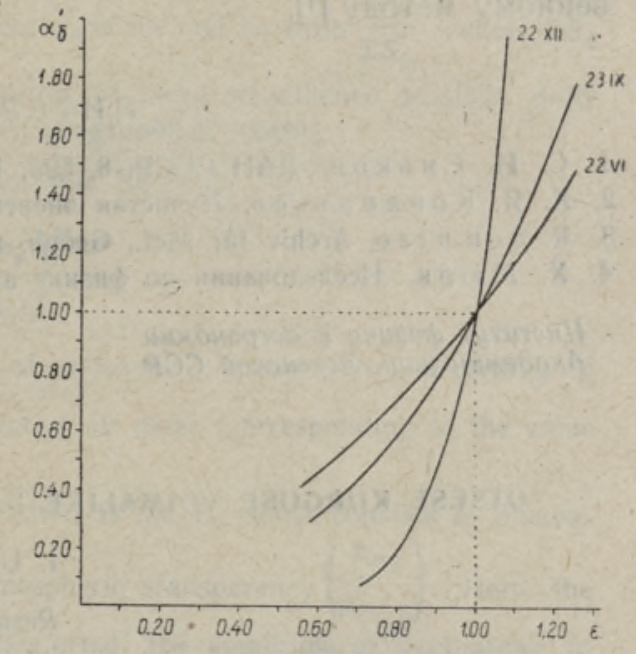

Рнс. 2. Зависимость относительных возможных дневных сумм инсоляции от эффективной прозрачности атмосферы $\left(\frac{p_{m_{\ni}}}{0,80}\right) ;$ географическая широта Тарту.

Формулу (6) можно рассматривать как формулу поправочного множителя, входящего в расчетную формулу

$$
\Sigma S_{6}^{\prime}=f\left(p_{m_{9}}\right) \Sigma S_{0}^{\prime},
$$

где $f\left(p_{m_{9}}\right)$ - функция от $p_{m_{9}}$. Но учитывая формулу (6), можно написать

$$
\Sigma S_{6}^{\prime}=\left(\frac{\dot{p}_{m_{9}}}{p_{m_{9,0}}}\right)^{m_{9,0}} \Sigma S_{0}^{\prime} .
$$

Масса атмосферы - сложная функция (функция Бемпорада), а по упрощающим предположениям $m \approx \operatorname{cosec} h_{\odot}$. Но в таком случае $m_{9} \approx \operatorname{cosec} h_{9}$ и

$$
\Sigma S_{6}^{\prime} \approx\left(\frac{p_{m_{9}}}{p_{m_{9,0}}}\right)^{\operatorname{cosec} h_{9,0}} \Sigma S_{0}^{\prime} .
$$

Расчет конкретных возможных дневных сумм инсоляции, исходя из формулы (15), сводится к вычислению фактических эффективных коэффициентов прозрачности атмосферы, причем климатологические величины, входящие в формулу (15), должны быть, конечно, известны.

Значение $p_{m_{9}, 0}$ можно, исходя из значения $\Sigma S_{0}^{\prime}$, найти по номограмме, дающей зависимость возможных дневных сумм инсоляции от эффективной прозрачности атмосферы; такая номограмма аналогична той, которая представлена Х. Мюрком [ $\left.{ }^{4}\right]$.

Эффективную массу атмосферы $\left(m_{9}, 0\right)$ можно определить из формулы

$$
\Sigma S_{0}^{\prime}=p_{m_{3,0}}^{m_{3,0}} \Sigma I_{c}^{\prime}
$$


где $\Sigma I_{c}$ - дневная сумма инсоляции, соответствующая условиям солярного климата. Из формулы (17) следует, что

$$
m_{9,0}=\frac{\lg \Sigma S_{0}^{\prime}-\lg \Sigma I_{c}^{\prime}}{\lg p_{m_{9,0}}} .
$$

Что касается определения фактических эффективных коэффициентов прозрачности $\left(p_{m_{9}}\right)$, то эти коэффициенты могут быть вычислены по косвенному методу ['].

\section{Л И ТЕ Р А Т Р А}

1. С. И. С и в ков, ДАН СССР, $8,599,1951$.

2. К. Я. Кондратьев, Лучистая энергия Солнца. Гидрометиздат. Л., 1954.

3. R. Sch ulze, Archiv für Met., Geoph. und Bioklimatol., S. B, 11, 281, 1962.

4. Х. М юр к, Исследования по физнке атмосферы. ИФА АН ЭССР, 4, 5, 1963.

\section{Институт физики и астрономии} Академии маук Эстонской ССР

Поступила в редакшию 30. III 1963

\section{OTSESE KIIRGUSE VOIMALIKE PAEVASUMMADE VARIEERUMISEST}

\section{Undla}

\section{Resümee}

Käesolev artikkel käsitleb Päikeselt horisontaalpinnale langeva otsese kiirguse võimalike päevasummade muutlikkuse küsimust. Oldjuhul ilmneb nimetatud varieerumine suhteliste summade $\left(a_{6}^{\prime}\right)$. köikumises. Siin defineeritakse suhtena $\frac{\Sigma S_{6}^{\prime}}{\Sigma S_{0}^{\prime}}$, kus $\Sigma S_{6}^{\prime}$ on vaadeldav võimalik päevasumma, $\Sigma S_{0}^{\prime}-$ keskmine tasandatud võimalik päevasumma (vastavalt kaléndripäevale ja geograafilisele punktile).

$\boldsymbol{a}_{6}^{\prime}$ võib avaldada valemiga $(6)$, nimelt

$$
\alpha_{6}^{\prime}=\left(\frac{p_{m_{9}}}{p_{m_{3,0}}}\right)^{m_{3,0}}
$$

kus $p_{m_{9}}$ ja $p_{m_{9,0}}$ on atmosfääri läbipaistvuse efektiivsed koefitsiendid (vastavalt $\Sigma S_{6}^{\prime}$-le ja $\Sigma S_{0}^{\prime}$-le), $m_{9,0}$ aga atmosfăäri efektiivne mass (vastavalt $\Sigma S_{0}^{\prime}$-le).

$\boldsymbol{a}_{6}^{\prime}$ väärtuste varieerumise pōhjus, vastavalt valemile (6), peitub atmosfääri läbipaistvuse suhtelise efektiivse koefitsiendi $\left(\frac{p_{m_{3}}}{p_{m_{9,0}}}\right)$ kōikumises; atmosfääri efektiivse massi kōikumisi vōib siinjuures mitte arvestada. $\alpha_{6}^{\prime}$ väärtuste kõikumise amplituud aga sõltub nii suhtest $\frac{p_{m_{9}}}{p_{m_{9,0}}}$ kui ka koefitsiendist $m_{9,0}$.

Arvitused näitavad, et $\boldsymbol{a}_{6}^{\prime}$ sōltub atmosfääri efektiivse massi aastasest käigust (järelikult ka geograafilisest) küllalt märkimisvăärselt (joon. 1). Atmosfääri erinevatele efektiivsetele massidele vastavate $\boldsymbol{\alpha}_{\sigma}^{\prime}$ väărtuste vōrdlemiseks on vaja suhtelised summad taandada ühele ja samale atmosfääri efektiivsele massile, s. o. praktiliselt ühele ja samale Păikese efektiivsele kõrgusele. 


\section{VARIATIONS IN POSSIBLE DAILY TOTALS OF DIRECT RADIATION}

\section{Undla}

\section{Summary}

The paper deals with the variability of the possible daily sums of direct solar radiation upon a horizontal plane. In a general way, the variation mentioned above is revealed in the fluctuations of the relative totals $\left(\alpha_{\sigma}^{\prime}\right)$. Here $\alpha_{6}^{\prime}$ is defined as ratio $\frac{\Sigma S_{6}^{\prime}}{\Sigma S_{0}^{\prime}}$, where $\Sigma S_{6}^{\prime}$ is the observed possible daily total. $\Sigma S_{0}^{\prime}$ represents the mean smoothened possible daily total corresponding to a given date and a given geographical locality.

The value of $\alpha_{6}^{\prime}$ may be expressed by the formula (6), i. e.

$$
=\alpha_{6}^{\prime}=\left(\frac{p_{m_{9}}}{p_{m_{9,0}}}\right)^{m_{9,0}},
$$

where $p_{m_{9}}$ and $p_{m_{3,0}}$ are effective coeificients of atmospheric transparency, according to the values of $\Sigma S_{6}^{\prime}$ and $\Sigma S_{0}^{\prime}$, and $m_{9,0}$ is the effective air mass (corresponding to the value of $\left.\Sigma S_{0}^{\prime}\right)$.

According to formula (6), the cause of variations in the $\alpha_{6}^{\prime}$ values consists in fluctuations of the relative effective coefficient of atmospheric transparency $\left(\frac{p_{m_{9}}}{p_{m_{3}, 0}}\right)$. Here the fluctuations of the effective air mass may be neglected. The amplitude of fluctuations in the $\alpha_{6}^{\prime}$ values depends on the values of $\frac{p_{m_{9}}}{p_{m_{9}, 0}}$, as well as on the values of $m_{9,0}$.

Calculations show that the $\alpha_{6}^{\prime}$ values are to a considerable extent dependent on the seasonal trend of the effective air mass (hence also on the geographical locality) (fig. 1). In order to compare the $\alpha_{6}^{\prime}$ values which correspond to the different values of effective air masses, the relative totals should be reduced to one and the same effective air mass, i. e. practically to the same effective altitude of the Sun.

Academy of Sciences of the Estonian S. S. R., Received Institute of Physics and Astronomy 